\title{
Underdetermined Direction-of-Arrival Estimation with Coprime Array via Atomic Norm Minimization
}

\author{
Yujian PAN ${ }^{1,2,3}$, Min YAO ${ }^{1}$, Guo Qing $L U O^{1}$, Bai Cao PAN ${ }^{1,2}$, Xiaoxin $G A O^{1}$ \\ ${ }^{1}$ Key Laboratory of RF Circuits and System, Ministry of Education, Institute of Antennas and Microwave Technology, \\ Hangzhou Dianzi University, 310018 Hangzhou, China \\ ${ }^{2}$ State Key Laboratory of Millimeter Waves, Southeast University, 210096 Nanjing, China \\ ${ }^{3}$ Dept. of Electrical Engineering, KU Leuven, 3001 Leuven, Belgium
}

pyj@hdu.edu.cn, luoguoqing@hdu.edu.cn

Submitted November 29, 2019/ Accepted March 11, 2020

\begin{abstract}
The coprime array provides the possibility of resolving more signals than the sensors for the directionof-arrival (DOA) estimation application. However, the non-consecution of its virtual array raises challenges for making full use of the degree of freedom (DOF). In this paper, we propose a new underdetermined DOA estimation method with coprime array where the non-consecutive virtual array can be converted into a virtual uniform linear array (ULA) with the same aperture. Firstly, all elements in the vectorized signal covariance matrix corresponding to the same virtual array positions are averaged to construct the output signals of the virtual array. Then, an atomic norm minimization (ANM) based optimization problem is formed for denoising the output signals of the virtual array and for interpolating the missing signals at the virtual array holes. At last, the ANM problem is solved by the semidefinite programming $(S D P)$ and the DOAs are obtained by applying the subspace method on the reconstructed signal covariance matrix of the interpolated virtual ULA. The proposed algorithm is gridless and makes full use of the DOF and the information provided by the coprime array. The simulation results compared with the other representative methods are given to demonstrate the superiority of the proposed method with respect to the resolution and estimation accuracy.
\end{abstract}

\section{Keywords}

Atomic norm minimization (ANM), coprime array, denoising and interpolating, gridless method, underdetermined direction-of-arrival (DOA) estimation

\section{Introduction}

Direction-of-arrival (DOA) estimation which is the technology utilizing multi-antenna array to estimate the DOAs of the waves is widely applied in military and civil fields, such as radar, electronic countermeasures, seismic exploration, and communication [1]. The ULA has been widely used due to its simple array configuration [2]. However, too much redundancy exists in the covariance domain of ULA. To reduce the redundancy of ULA and to provide possibility for resolving more signals than sensors, some sparse arrays are proposed. The minimum redundancy array (MRA) [3] and minimum hole array (MHA) [4] are the old ones. However, they do not have a systematic array structures and they need to be determined by exhaustive tests. In contrast, the newly proposed coprime array [5] and the nested array [6] have a closed-form expression for the array configuration. Among them, the coprime array receives more attention since it is less affected by the mutual coupling.

Coprime array, as a kind of sparse array, can provide $\mathcal{O}\left(M^{2}\right)$ DOFs only using $M$ physical elements. However, the coprime array is a partially augmentable array [7], which means the virtual array derived from coprime array is non-consecutive. This leads to the fact that the ULAbased methods cannot be directly applied to the coprime array. In order to use the ULA-based methods, a common practice is to only employ the longest consecutive subarray of virtual array for DOA estimation [8]. The drawbacks of this method are the information loss and the underutilization of DOF. Although the low rank matrix denoising algorithm in [9] uses the low rank property of the covariance matrix to reduce the DOA estimation error caused by noise, it still has not made full use of the DOF.

For this problem, compressed sensing [10], [11] provides a new solution since this kind of methods can theoretically deal with arbitrary array. The least absolute shrinkage and selection operator (LASSO) based algorithm in [12] is an example. Unfortunately, another problem called basis mismatch arises [13]. Since the sparse dictionary in compressed sensing is discrete, the estimated DOAs can only be located at the pre-defined grids. In fact, the true DOAs cannot be exactly on grids. In order to solve this problem, the off-grid method is proposed [14]. Nevertheless, the basis mismatch problem has not been fully solved until the gridless methods were proposed. 
The gridless methods in [15]-[22] can estimate parameters without setting grid points in advance. For the coprime array application, a nuclear norm minimizationbased method was proposed in [21]. The missing signals in virtual array are interpolated by the concept of matrix completion. However, there is no denoising operation in this method. The virtual array interpolation-based method is also proposed in [22], which makes full use of the DOF. Nevertheless, this method has not fully utilized the signal information due to discarding some elements in covariance matrix and this method forms an optimization problem in the pattern of multiple measurement vectors [23], leading to high computational complexity.

In this paper, we propose a new underdetermined DOA estimation method with coprime array. Through interpolating the non-consecutive virtual array to virtual ULA, we can make full use of the DOF. Through averaging all elements in the vectorized signal covariance matrix corresponding to the same virtual array positions, we utilize all the signal information successfully. The interpolation operation is performed by forming and solving an ANM based problem where the denoising operation is also involved. Since ANM belongs to the gridless compressed sensing, the basis mismatch effect is avoided in this method. The ANM problem is solved by SDP and the DOAs are obtained by applying the subspace method on the reconstructed signal covariance matrix of the interpolated virtual ULA. Simulation results demonstrate the superiority of the proposed method in terms of resolution and estimation accuracy.

The rest paper is organized as follows. Section 2 revisits coprime signal model. Section 3 presents the proposed method that contains the details of processing. The numerical simulation results are given in Sec. 4. Finally, Section 5 concludes this paper.

Notations: $\mathbb{C}$ denotes the set of complex number. $(\cdot)^{\mathrm{T}},(\cdot)^{*}$ and $(\cdot)^{\mathrm{H}}$ denote the transpose, conjugate, and conjugate transpose, respectively. $\|\cdot\|_{2}$ represents the $\ell_{2}$ norm. $\otimes$ stands for the Kronecker product. $\lambda$ is the wavelength of the signal. $\mathcal{F}$ denotes the DOF of the array. The notation $\mathrm{E}[\cdot]$ represents the statistical expectation. $\operatorname{vec}(\cdot)$ represents the vectorizing operator. $\operatorname{Tr}(\cdot)$ denotes the trace of the matrix. $|\mathcal{S}|$ denotes the cardinality of a set $\mathcal{S}$.

\section{Coprime Signal Model}

For the coprime array, there are several types of configurations [24]. In this paper, we choose the so called extended coprime array proposed in [8] for example and the method proposed in this paper is also applicable for other types of coprime array. The extended coprime array is composed of a pair of ULAs. In the case of $M<N$, one subarray consists of $2 M$ sensors spaced $N d$ apart and the position of the array elements can be expressed as $\mathcal{S}_{1} d$, where $\mathcal{S}_{1}=\{m N, 0 \leq m \leq 2 M-1\}$ and $d$ is a half-wavelength

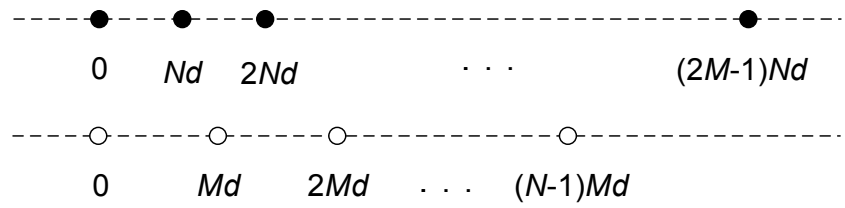

Fig. 1. The extended coprime array configuration.

i.e., $d=\lambda / 2$. The other one consists of $N$ sensors spaced $M d$ apart and the position of the array elements can be expressed as $\mathcal{S}_{2} d$, where $\mathcal{S}_{2}=\{n M, 0 \leq n \leq N-1\}$. Here, $M$ and $N$ satisfy the coprime condition. The coprime array $\mathcal{S}$ is formed by overlapping the first array element of the two subarrays, as showed in Fig. 1. Thus, this coprime array consists of $N+2 M-1$ sensors in total and its DOF can be up to $\mathcal{F}=3 M N+M-N=\mathcal{O}(M N)$ [21].

Supposed that there are $K$ far-field narrow-band plane wave signals with DOA $\boldsymbol{\theta}=\left[\theta_{1}, \theta_{2}, \ldots, \theta_{K}\right]^{\mathrm{T}}$ impinging at the coprime array $\mathcal{S}$, and then the output signal of this array at time $t$ is

$$
\boldsymbol{x}(t)=\sum_{k=1}^{K} \boldsymbol{a}\left(\theta_{k}\right) \boldsymbol{s}_{k}(t)+\boldsymbol{n}(t)=\boldsymbol{A s}(t)+\boldsymbol{n}(t)
$$

where $\boldsymbol{A}=\left[\boldsymbol{a}\left(\theta_{1}\right), \boldsymbol{a}\left(\theta_{2}\right), \ldots, \boldsymbol{a}\left(\theta_{K}\right)\right] \in \mathbb{C}^{(N+2 M-1) \times K}$ stands for the manifold matrix of the coprime array. $\boldsymbol{s}(t)=\left[\boldsymbol{s}_{1}(t), \boldsymbol{s}_{2}(t), \ldots, \boldsymbol{s}_{K}(t)\right]^{\mathrm{T}}$ denotes the signal waveform vector and $\boldsymbol{n}(t)=\left[\boldsymbol{n}_{1}(t), \boldsymbol{n}_{2}(t), \ldots, \boldsymbol{n}_{K}(t)\right]^{\mathrm{T}}$ denotes the additive white Gaussian noise component which is independent with the incident signal waveform vector. $\boldsymbol{a}\left(\theta_{k}\right)$ represents the steering vector of the $k$ th source, which can be expressed as

$$
\boldsymbol{a}\left(\theta_{k}\right)=\left[1, \exp \left(-\mathrm{j} \xi u_{2} \cos \left(\theta_{k}\right)\right), \ldots, \exp \left(-\mathrm{j} \xi u_{N+2 M-1} \cos \left(\theta_{k}\right)\right)\right]^{\mathrm{T}}
$$

where $\xi=2 \pi d / \lambda$ and $\mathrm{j}=\sqrt{-1}$ is the imaginary unit. $u_{k}$ denotes the position of the $k$ th physics sensor with $u_{1}=0$.

\section{The Proposed DOA Estimation Algorithm}

\subsection{The Formation of Virtual Array and Its Output Signal}

The output signal covariance matrix can be expressed as

$$
\boldsymbol{R}_{\boldsymbol{x}}=\mathrm{E}\left[\boldsymbol{x}(t) \boldsymbol{x}(t)^{\mathrm{H}}\right]=\boldsymbol{A} \mathrm{E}\left[\boldsymbol{S} \boldsymbol{S}^{\mathrm{H}}\right] \boldsymbol{A}^{\mathrm{H}}+\sigma_{n}^{2} \boldsymbol{I}
$$

where $\boldsymbol{R}_{\boldsymbol{S}}=\mathrm{E}\left[\boldsymbol{S} \boldsymbol{S}^{\mathrm{H}}\right]$ denotes the covariance matrix of sources and it is a diagonal matrix. $\sigma_{n}^{2}$ denotes the power of noise. I stands for the $(N+2 M-1) \times(N+2 M-1)$ identity matrix. In practice, the covariance matrix of the output signal is calculated by the following formula 


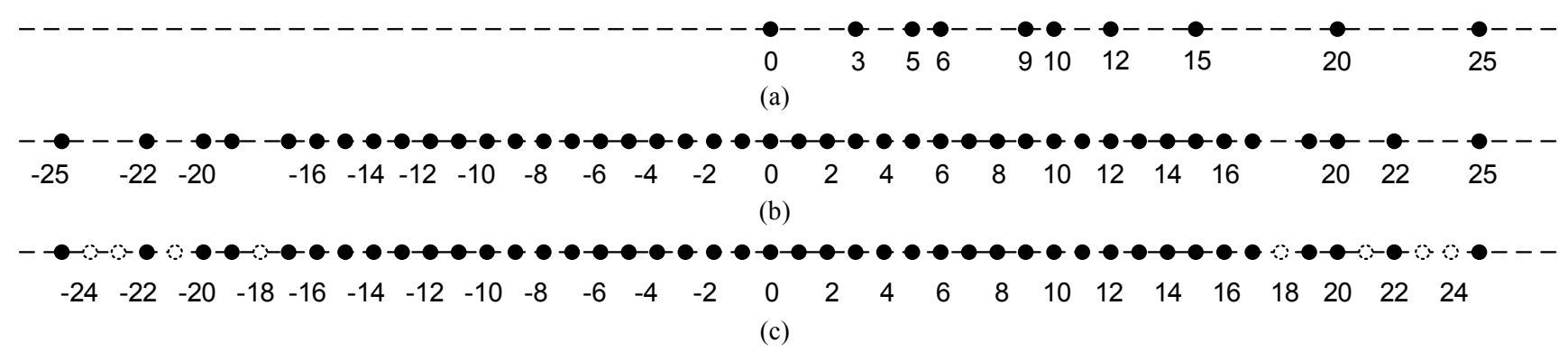

Fig. 2. Illustration of coprime array with $M=3$ and $N=5$ : (a) Extended coprime array configuration; (b) Virtual array derived from this coprime array; (c) Interpolated virtual ULA.

$$
\hat{\boldsymbol{R}}_{\boldsymbol{x}}=\frac{1}{T} \sum_{t=1}^{T} \boldsymbol{x}(t) \boldsymbol{x}(t)^{\mathrm{H}}
$$

where $T$ denotes the number of snapshots. When $T$ increases infinitely, the estimated $\hat{\boldsymbol{R}}_{x}$ infinitely approximates the true $\boldsymbol{R}_{\boldsymbol{x}}$.

The output signals of the virtual array can be constructed from the second order statistics of received signal. Vectorizing the covariance matrix, we have

$$
\boldsymbol{y} \triangleq \operatorname{vec}\left(\hat{\boldsymbol{R}}_{x}\right)=\sum_{k=1}^{K} \boldsymbol{v}\left(\theta_{k}\right) p_{k}+\sigma_{n}^{2} \boldsymbol{i}
$$

where $\boldsymbol{v}\left(\theta_{k}\right)=\boldsymbol{a}^{*}\left(\theta_{k}\right) \otimes \boldsymbol{a}\left(\theta_{k}\right), p_{k}$ denotes the power of the $k$ th source and $\boldsymbol{i}=\operatorname{vec}(\boldsymbol{I})$. The position of the virtual array is $\mathcal{S}_{\mathcal{D}} d$ and

$$
\mathcal{S}_{\mathcal{D}}=\left\{u_{m}-u_{n} \mid m, n=0,1, \ldots, N+2 M-1\right\}
$$

where $\left|\mathcal{S}_{\mathcal{D}}\right|=3 M N+M-N$. Since the coprime array is a partially augmentable array, the virtual array derived from coprime array has several missing elements that are referred to as the holes. For the sake of intuition, we give an example of extended coprime array with $M=3$ and $N=5$ in Fig. 2. Obviously, the virtual array shown in Fig. 2(b) is non-consecutive. The dotted circles in Fig. 2(c) indicate the positions of the holes which are at $\{ \pm 18, \pm 21$, $\pm 23, \pm 24\}$. Figure 2(c) is called the interpolated virtual array which will be used in the following.

By averaging the elements in $\boldsymbol{y}$ corresponding to the same positions in $\mathcal{S}_{\mathcal{D}}$, output signals of the virtual array $\tilde{\boldsymbol{y}}$ can be obtained as

$$
\tilde{\boldsymbol{y}}=\tilde{\boldsymbol{V}} \boldsymbol{p}+\sigma_{n}^{2} \tilde{\boldsymbol{I}}
$$

where $\tilde{\boldsymbol{V}} \in \mathbb{C}^{\left|\mathcal{S}_{D}\right| \times K}$ denotes the virtual array manifold matrix. $\boldsymbol{p}$ is the signal power vector and $\tilde{\boldsymbol{I}}$ is a vector that only the middle entry is 1 and the others are 0 . Here, by the averaging operation, the whole signal information provided by coprime array can be fully utilized.

Since the virtual array derived from coprime array has some holes, the virtual signals at the holes actually do not exist. However, we can assume there are imaginary ele- ments at the holes and the output signals of the imaginary elements can be interpolated later. Combined with the imaginary elements, the non-consecutive virtual array will be a virtual ULA with sensors positions at $\mathcal{S}_{\mathcal{V}} d$ where $\mathcal{S}_{\mathcal{V}}$ is the interpolated consecutive version of $\mathcal{S}_{\mathcal{D}}$, just like the array in Fig. 2(c) and $\left|\mathcal{S}_{\mathcal{V}}\right|=4 M N-2 N+1$. So, in this way, we can employ all the DOF provided by the coprime array. By initializing the signals of the imaginary elements to zero, we can form the output signals of the virtual ULA as

$$
\langle z\rangle_{i}= \begin{cases}\langle\tilde{\boldsymbol{y}}\rangle_{i}, \quad i \in \mathcal{S}_{\mathcal{D}} \\ 0, \quad i \in \mathcal{S}_{\mathcal{V}} \backslash \mathcal{S}_{\mathcal{D}}\end{cases}
$$

where \langle\rangle$_{i}$ represents the signal of the array element at position $i d$.

\subsection{Interpolating and Denoising Signals of Virtual ULA Based on ANM}

The concept of atomic norm and many common sparse norms such as $\ell_{1}$ norm, $\ell_{2}$ norm, and the nuclear norm of matrices are introduced in [25]. An atom for representing $z$ can be defined as

$$
\mathcal{A}=\left\{\tilde{\boldsymbol{v}}\left(\theta_{k}\right), \theta_{k} \in\left[0^{\circ}, 180^{\circ}\right)\right\} .
$$

The atomic norm of the interpolated output signal $z$ is defined as the smallest number of atoms in $\mathcal{A}$ that can express $z$

$$
\begin{aligned}
& \|z\|_{\mathcal{A}}=\inf \{t>0: z \in t \operatorname{conv}(\mathcal{A})\} \\
& =\inf \left\{\sum_{k} p_{k}: z=\sum_{k} p_{k} \tilde{\boldsymbol{v}}\left(\theta_{k}\right), p_{k} \geq 0, \theta_{k} \in\left[0^{\circ}, 180^{\circ}\right)\right\}
\end{aligned}
$$

where inf denotes the infimum. Therefore, the optimal $z^{*}$ can be obtained by solving the following ANM problem

$$
\min _{z} \| z_{\boldsymbol{A}}, \text { s.t. }\left\|z_{\Omega}-z_{\Omega}^{0}\right\|_{2}^{2} \leq \eta^{2}
$$

where $\eta^{2}$ denotes the upper limit of noise variance. $\mathbf{\Omega}$ represents the elements positions of non-consecutive 
virtual array. For example, $\boldsymbol{\Omega}$ can be expressed as a set of array element positions in Fig. 2(b), i.e. $\boldsymbol{\Omega}=\{0, \pm 1, \pm 2, \ldots$, $\pm 17, \pm 19, \pm 20, \pm 22, \pm 25\} . \quad \boldsymbol{z}_{\Omega}^{0}=\tilde{\boldsymbol{y}}$. Then atomic norm $\|z\|_{\mathcal{A}}$ can be converted into the following semidefinite programming (SDP) (The proof can be found in [26]).

$$
\|\|_{\mathcal{A}}=\inf \left\{\frac{1}{2\left|\mathcal{S}_{\mathcal{V}}\right|} \operatorname{Tr}(\mathrm{T}(\boldsymbol{u}))+\frac{1}{2} t \mid\left[\begin{array}{cc}
t & \boldsymbol{z}^{\mathrm{H}} \\
\boldsymbol{z} & \mathrm{T}(\boldsymbol{u})
\end{array}\right] \geq 0\right\}
$$

where $\mathrm{T}(\boldsymbol{u}) \in \mathbb{C}^{\left|\mathcal{S}_{v}\right| x\left|\mathcal{S}_{v}\right|}$ denotes a Hermitian Toeplitz matrix with vector $\boldsymbol{u} \in \mathbb{C}^{\left|\mathcal{S}_{v}\right|}$ as its first column. Therefore, the atomic norm $\|z\|_{\mathcal{A}}$ can be calculated by the following formula

$$
\min _{\boldsymbol{z}, \boldsymbol{u}} \operatorname{Tr}(\mathrm{T}(\boldsymbol{u}))+w, \text { s.t. }\left[\begin{array}{cc}
\boldsymbol{w} & \boldsymbol{z}^{\mathrm{H}} \\
\boldsymbol{z} & T(\boldsymbol{u})
\end{array}\right] \geq 0
$$

where $w=\left|\mathcal{S}_{\mathcal{V}}\right| t$ and $t$ is a turning parameter. It follows from (13) that the formula (11) can be cast as the following SDP problem

$$
\min _{z, \boldsymbol{u}} \operatorname{Tr}(\mathrm{T}(\boldsymbol{u}))+w, \text { s.t. }\left[\begin{array}{cc}
\boldsymbol{w} & \boldsymbol{z}^{\mathrm{H}} \\
\boldsymbol{z} & T(\boldsymbol{u})
\end{array}\right] \geq 0,\left\|\boldsymbol{z}_{\boldsymbol{\Omega}}-\boldsymbol{z}_{\mathbf{\Omega}}^{0}\right\|_{2}^{2} \leq \eta^{2} .
$$

According to the Lagrangian analysis, the dual problem of the (14) can be expressed as the following form (The proof is given in the Appendix A [27].)

$$
\begin{aligned}
& \min _{\boldsymbol{v}, \boldsymbol{W}} \eta\left\|\boldsymbol{v}_{\boldsymbol{\Omega}}\right\|_{2}+\mathfrak{R} e\left(\boldsymbol{z}_{\boldsymbol{\Omega}}^{0 \mathrm{H}} \boldsymbol{v}_{\boldsymbol{\Omega}}\right), \\
& \text { s.t. }\left[\begin{array}{ll}
1 & \boldsymbol{v}^{\mathrm{H}} \\
\boldsymbol{v} & \boldsymbol{W}
\end{array}\right] \geq 0, \boldsymbol{v}_{\overline{\mathbf{\Omega}}}=0, \mathrm{~T}^{*}(\boldsymbol{W}-\boldsymbol{I})=0
\end{aligned}
$$

where $\eta$ is the regularization parameter, $\mathfrak{R} e(\cdot)$ indicates taking the real value and $\mathrm{T}^{*}(\cdot)$ denotes the adjoint operator of $\mathrm{T}(\cdot)$. By using standard solver SDPT3 [28], the dual problem (15) can be solved more efficiently than the primal problem (14). According to the duality, the Lagrange multiplier corresponding to the first constraint of (15) is exactly the matrix in the first constraint of (14) which contains $\boldsymbol{z}$. So, after the formula (15) is solved, the interpolated output signal $\boldsymbol{z}^{*}$ can also be obtained.

\subsection{Covariance Matrix Reconstruction for DOA Estimation}

Since the interpolated output signal $z^{*}$ is a rank-1 signal, this problem is equivalent to the DOA estimation of coherent signal or single snapshot. Although spatial smoothing operation is capable of removing the coherence of the signal, we choose a more efficient method proposed in [29] to reconstruct the rank-K signal covariance matrix. Setting $L=\left(\left|\mathcal{S}_{\mathcal{V}}\right|+1\right) / 2$, we can reconstruct the covariance matrix $\tilde{\boldsymbol{R}}_{\boldsymbol{v}}$ as

$$
\tilde{\boldsymbol{R}}_{\boldsymbol{v}}=\left[\begin{array}{cccc}
\boldsymbol{z}_{L}^{\star} & \boldsymbol{z}_{L-1}^{\star} & \cdots & \boldsymbol{z}_{1}^{\star} \\
\boldsymbol{z}_{L+1}^{\star} & \boldsymbol{z}_{L}^{\star} & \cdots & \boldsymbol{z}_{2}^{\star} \\
\vdots & \vdots & \ddots & \vdots \\
\boldsymbol{z}_{2 L-1}^{\star} & \boldsymbol{z}_{2 L-2}^{\star} & \cdots & \boldsymbol{z}_{L}^{\star}
\end{array}\right] .
$$

After the signal covariance matrix is reconstructed, the DOAs can be obtained by the subspace method such as the MUSIC [8], [29], [30], the ESPRIT [31]-[33], and the root-MUSIC [34], [35]. Here, we describe the MUSIC spectrum as following formula

$$
P_{\text {MUSIC }}(\theta)=\frac{1}{\boldsymbol{a}_{\mathrm{s}}^{\mathrm{H}}(\theta) \boldsymbol{U}_{\mathrm{N}} \boldsymbol{U}_{\mathrm{N}}^{\mathrm{H}} \boldsymbol{a}_{\mathrm{s}}(\theta)}
$$

where $\boldsymbol{a}_{\mathrm{s}}^{\mathrm{H}}(\theta)$ is the steering vector of sub virtual ULA from the position of 0 to $L d$. $\boldsymbol{U}_{\mathrm{N}}$ is the noise subspace. The DOAs are found by searching the locations of the $K$ largest peaks of the spectrum.

The proposed algorithm is described in Algorithm 1. The advantages of this method are summarized as follows. Firstly, the non-consecutive virtual array is interpolated to the virtual ULA, so we make full use of the DOF. Secondly, by averaging all elements in the vectorized signal covariance matrix corresponding to the same virtual array positions, we utilized all the signal information. Furthermore, in this optimization procedure, the denoising operation is involved, which increases the accuracy of the estimation. At last, the gridless ANM based optimization method avoids the basis mismatch problem.

\section{Algorithm 1 Virtual Array Interpolation-Based Underdetermined DOA Estimation via Atomic Norm Minimization}

1. Input: Extended coprime array received signal $\{\boldsymbol{x}(t)\}_{t=1}^{T}$ and the number of sources $K$.

2. Output: $\hat{\theta}_{k}, k=1,2, \ldots, K$.

3. Calculate the covariance matrix $\hat{\boldsymbol{R}}_{x}$ and construct the output signal $\tilde{\boldsymbol{y}}$ corresponding to the nonconsecutive virtual array $\mathcal{S}_{\mathcal{D}}$.

4. Initialize the interpolated output signal $\boldsymbol{z}$ via (8).

5 . Restore the interpolated output signal $z^{*}$ with respect to interpolated virtual ULA $\mathcal{S}_{\mathcal{V}}$ via (15).

6. Reconstruct the signal covariance matrix $\tilde{\boldsymbol{R}}_{v}$ of the interpolated virtual ULA via (16).

7. Estimate the spatial spectrum by using MUSIC algorithm in (17) to get the DOA estimation. 


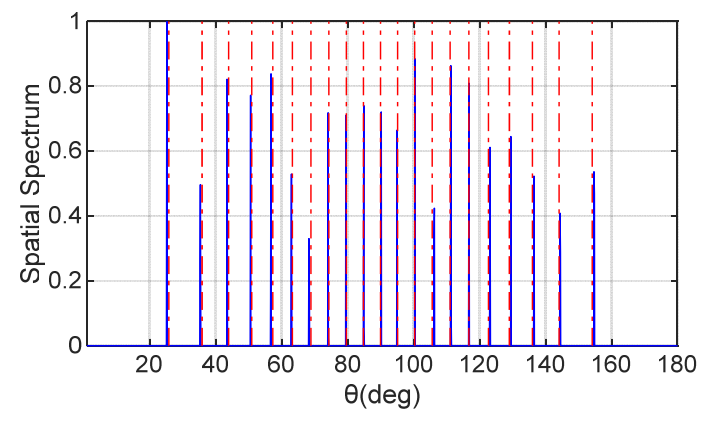

(a)

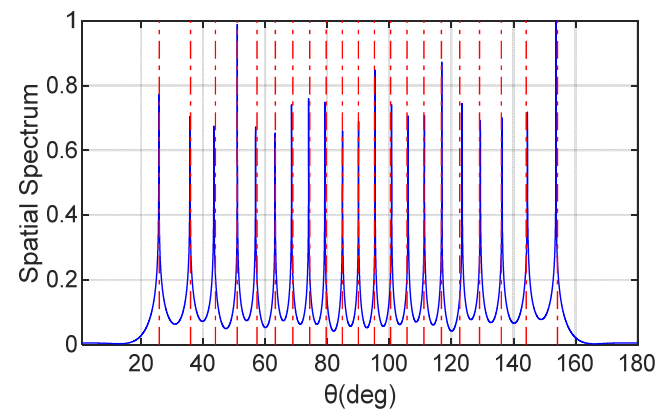

(c)

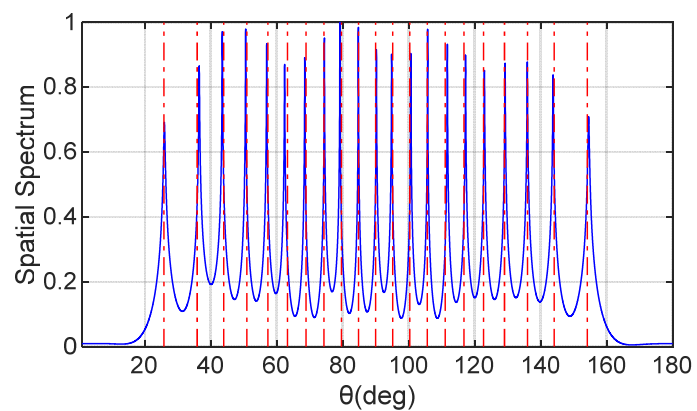

(b)

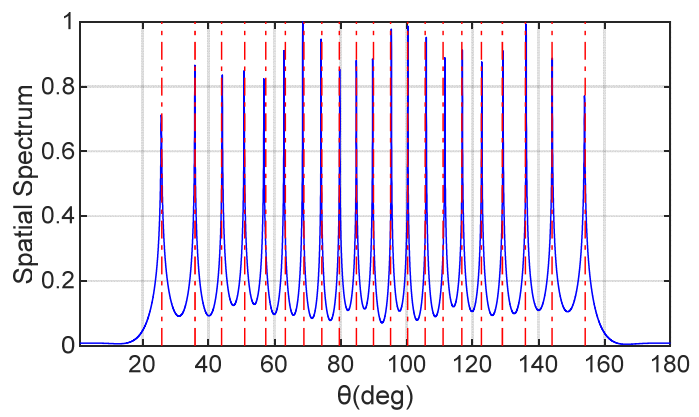

(d)

Fig. 3. The spatial spectrum of these algorithms. The number of sources is 21 . The vertical dashed lines denote the actual DOA of the sources: (a) LASSO algorithm; (b) NNM algorithm; (c) CA-VAI algorithm; (d) Proposed algorithm.

\section{Numerical Experiments}

An extended coprime array with $M=3, N=5$ is adopted, which yields a total number of $N+2 M-1=10$ physical sensors located at $\{0,3 d, 5 d, 6 d, 9 d, 10 d, 12 d$, $15 d, 20 d, 25 d\}$, where $d$ is half wavelength. The proposed method is compared with several representative algorithms, namely the spatial smoothing method (SS-MUSIC) in [8], the low rank matrix denoising (LRD) in [9], the least absolute shrinkage and selection operator (LASSO) in [12] the nuclear norm minimization (NNM) in [21], and the virtual array interpolation-based (CA-VAI) in [22]. The regularization parameters for LASSO algorithm, LRD algorithm, CA-VAI algorithm, and the proposed algorithm are set as $1.5,10,0.25$, and 1 , respectively. The convex optimization problems in the above algorithms are all solved by the CVX package using MATLAB software [36].

\subsection{Achievable DOF}

In this section, we compare the achievable DOF of the proposed method with other algorithms. Supposed there are 21 uncorrelated sources whose cosine values of the source angle are uniformly distributed from -0.9 to 0.9 . As SS-MUSIC and LRD cannot handle so many sources, we only choose LASSO, NNM, and CA-VAI. The SNR and the number of snapshots are set as $10 \mathrm{~dB}$ and 400 , respectively. The grid interval of LASSO algorithm is set as $\Delta \theta=0.2^{\circ}$. It is showed in Fig. 3 that each algorithm can achieve effective resolution of 21 sources only using 10 physical array elements. We take 1000 Monte Carlo trials and measure their recovery accuracy with the root-meansquared error (RMSE) and represent it under each subgraph. The RMSE is defined as follows

$$
\mathrm{RMSE}=\sqrt{\frac{1}{K Q} \sum_{k=1}^{K} \sum_{q=1}^{Q}\left(\hat{\theta}_{k}(q)-\theta_{k}\right)^{2}}
$$

where $\hat{\theta}_{k}(q)$ is the DOA estimation in the $q$ th MonteCarlo trial. $Q$ denotes the total number of Monte-Carlo trials. We can see that the RMSE of the proposed algorithm is the smallest as shown in Fig. 3. This is because that LASSO can only estimate the DOA on predefined grid points, increasing the estimation error. Although NNM based on matrix completion can get the DOA, the signal has not been denoised, and the limited number of snapshots can affect the accuracy of matrix completion, resulting in an affect the accuracy of matrix completion, resulting in a large estimation error. CA-VAI and the proposed method both use an interpolation-based approach, but the CA-VAI has not fully utilized all the information provided by the covariance matrix. In contrast, the proposed method makes full use of the information provided by the signal and improves the estimation accuracy. 


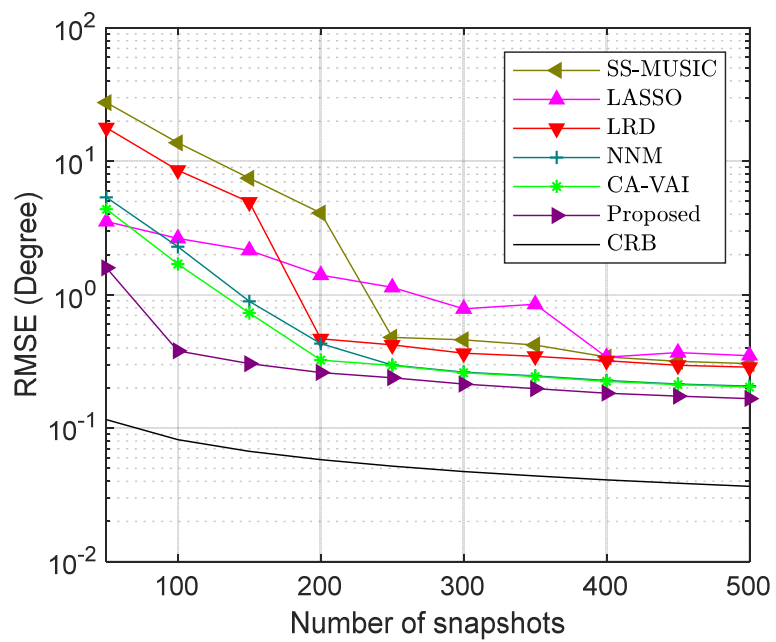

(a)

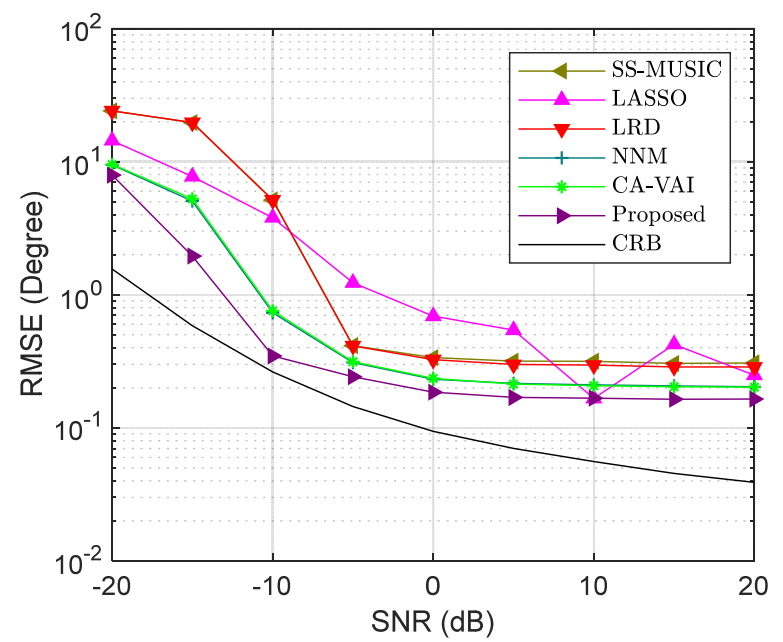

(b)

Fig. 4. RMSE performance comparison in each algorithm: (a) RMSE versus the number of snapshots with $\mathrm{SNR}=20 \mathrm{~dB}$. (b) RMSE versus SNR with the number of snapshots $T=500$.

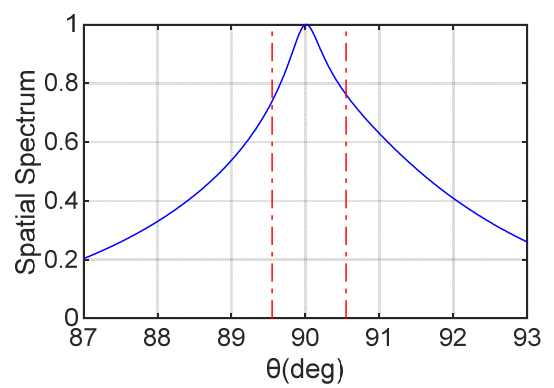

(a)

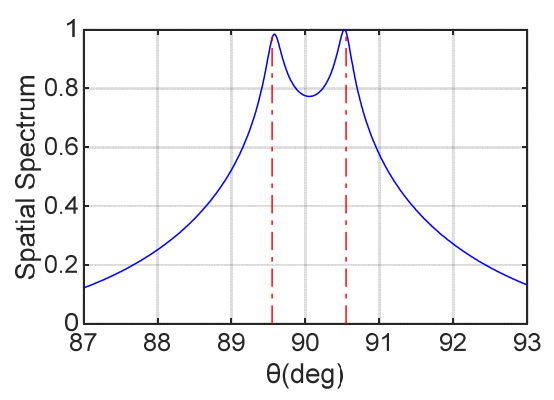

(d)

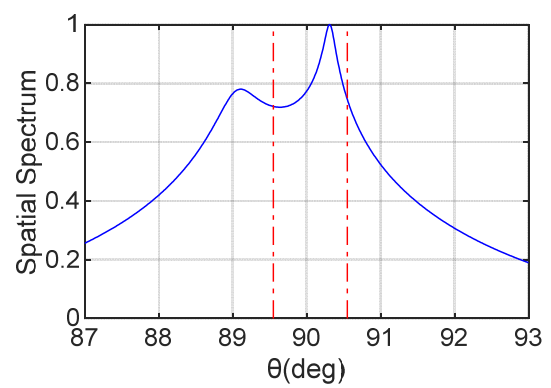

(b)

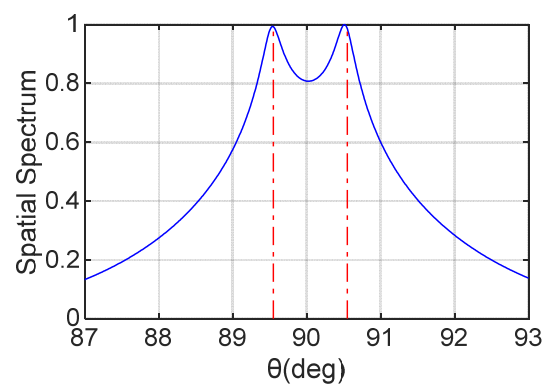

(e)

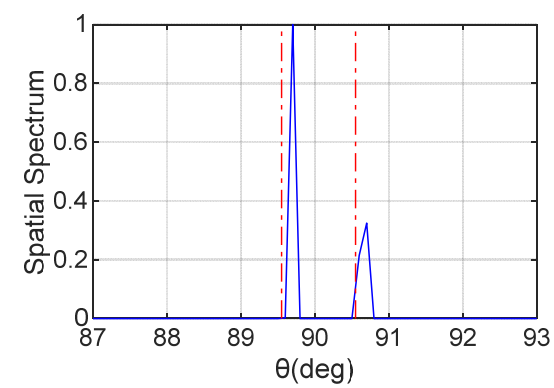

(c)

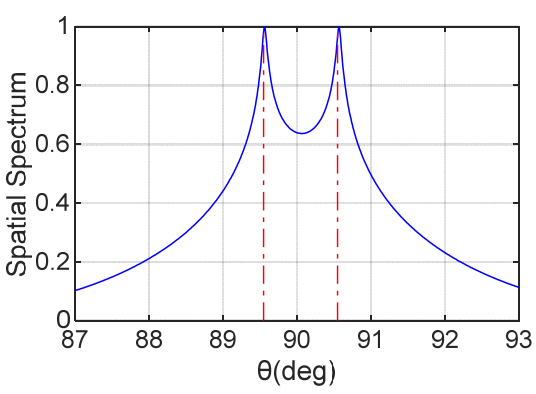

(f)

Fig. 5. DOA Resolution comparison of different algorithms. $T=400$ and $\mathrm{SNR}=10 \mathrm{~dB}$. The vertical dashed lines denote the actual DOA of sources: (a) SS-MUSIC algorithm; (b) LRD algorithm; (c) LASSO algorithm; (d) NNM algorithm; (f) CA-VAI algorithm; (f) Proposed algorithm.

\subsection{Estimation Performance Analysis}

In this section, we set the number of sources as 12 . The sources are uniformly spaced from $25^{\circ}$ to $155^{\circ}$ and each DOA is added with a random jitter which follows the uniform distribution in $\left[-0.1^{\circ}, 0.1^{\circ}\right]$ to reduce the impact of basis mismatch effect. The grid interval of LASSO algorithm is set as $\Delta \theta=0.1^{\circ} .1000$ Monte Carlo trials are run. The Cramér-Rao bound (CRB) [37] is also given as the reference.

The results of RMSE versus the number of snapshots is shown in Fig. 4(a), where the SNR is $20 \mathrm{~dB}$. It can be seen that the trend of the RMSE curves of various algorithms is consistent with the trend of the CRB curve. As for LASSO, it generates spurious peaks in the spectrum, leading to the fluctuation tendency of the curve. SS-MUSIC and LRD only use a part of array elements, leading to a relatively large RMSE. NNM is a method using all DOF, which makes its performance better than former two algorithms. CA-VAI achieves lower RMSE than NNM due to the involved denoising operation. The performance of the proposed algorithm outperforms significantly than other algorithms. Also, when the number of snapshots is greater than 100, the RMSE value of the proposed algorithm be- 
gins to decrease, indicating the performance threshold is obviously lower than other algorithms. A similar performance comparison is given by the simulation of the RMSE versus SNR for each algorithm in Fig. 4(b) with the number of snapshots $T=500$. Again, the results are similar to Fig. 4(a) and the proposed method outperforms others under all value of SNR.

\subsection{Resolution}

In this section, we are comparing the resolution performance of the proposed algorithm with other five algorithms. Two sources located at $89.55^{\circ}$ and $90.55^{\circ}$ are considered with the number of snapshots $T=400$ and $\mathrm{SNR}=10 \mathrm{~dB}$. The results are given in Fig. 5. It is showed that the SS-MUSIC algorithm is not able to resolve the sources and the resolution of LRD is very poor. This is because they only use partial array elements of the virtual array. LASSO can resolve the two sources, but the peak location is not accurate, which is because it is an on-grid estimator. The remaining three algorithms are gridless method and they all exhibit accurate peaks. However, among them, we find the peaks of the proposed method are the sharpest, indicating the best resolution performance.

\section{Conclusion}

In this paper, we proposed a new underdetermined DOA estimation method for the coprime array application. This algorithm makes full use of the array DOF. Through averaging all elements in the vectorized signal covariance matrix corresponding to the same virtual array positions, this algorithm also makes full use of the signal information. The optimization problem in this method is formed and solved under the framework of ANM, indicating it is a gridless method. Simulation results have shown the superiority of the proposed method in terms of resolution and estimation accuracy. Furthermore, this method can also be easily extended to ULA, MRA, nested array and other sparse arrays. Future works are to consider the scenarios of correlated and coherent signals and to perform DOA estimation with unknown number of sources.

\section{Acknowledgments}

This work was supported in part by the National Natural Science Foundation of China under Contract 61701145, Contract 61722107, in part by the Open Research Program of State Key Laboratory of Millimeter Waves under Grant No. K202034, No. K201912, in part by the scholarship from China Scholarship Council under the Grant CSC No. 201908330075 and in part by the Zhejiang Provincial Natural Science Foundation under Grant No. LQ18F010004. The authors would like to thank
Dr. C. Zhou from Zhejiang University, China, for discussions about the underdetermined CRB.

\section{Appendix A}

Let $\boldsymbol{\Lambda}=\left[\begin{array}{ll}s & \boldsymbol{v}^{\mathrm{H}} \\ \boldsymbol{v} & \boldsymbol{W}\end{array}\right] \geq 0$. The Lagrangian function of (14) is given as follows

$$
\begin{aligned}
\mathcal{L}(\boldsymbol{u}, w, \boldsymbol{z}, \boldsymbol{\Lambda}, \lambda)= & \operatorname{Tr}(\mathrm{T}(\boldsymbol{u}))+w-\operatorname{Tr}\left(\left[\begin{array}{cc}
w & \boldsymbol{z}^{\mathrm{H}} \\
\boldsymbol{z} & T(\boldsymbol{u})
\end{array}\right] \boldsymbol{\Lambda}\right) \\
& +\lambda\left\|z_{\boldsymbol{\Omega}}-\boldsymbol{z}_{\mathbf{\Omega}}^{0}\right\|_{2}^{2}-\lambda \eta^{2} \\
& =\operatorname{Tr}(\mathrm{T}(\boldsymbol{u}))+w-w s-2 \Re e\left(\boldsymbol{z}^{\mathrm{H}} \boldsymbol{v}\right)-\operatorname{Tr}(\mathrm{T}(\boldsymbol{u}) \boldsymbol{W}) \\
& +\lambda\left\|z_{\boldsymbol{\Omega}}-\boldsymbol{z}_{\mathbf{\Omega}}^{0}\right\|_{2}^{2}-\lambda \eta^{2} \\
& =\operatorname{Tr}[\mathrm{T}(\boldsymbol{u})(\boldsymbol{I}-\boldsymbol{W})]+w(1-s)-2 \Re e\left(\boldsymbol{z}^{\mathrm{H}} \boldsymbol{v}\right) \\
& -\lambda^{-1}\left\|\boldsymbol{v}_{\mathbf{\Omega}}\right\|_{2}^{2}+\lambda\left\|\boldsymbol{z}_{\mathbf{\Omega}}-\boldsymbol{z}_{\mathbf{\Omega}}^{0}-\lambda^{-1} \boldsymbol{v}_{\mathbf{\Omega}}\right\|_{2}^{2}-\lambda \eta^{2} \\
& +2 \Re e\left(\boldsymbol{z}_{\mathbf{\Omega}}^{\mathrm{H}} \boldsymbol{v}_{\mathbf{\Omega}}\right)-2 \Re e\left(z_{\mathbf{\Omega}}^{0 \mathrm{H}} \boldsymbol{v}_{\mathbf{\Omega}}\right) \\
& =\operatorname{Tr}[\mathrm{T}(\boldsymbol{u})(\boldsymbol{I}-\boldsymbol{W})]+w(1-s)-2 \Re e\left(\boldsymbol{z}_{\overline{\mathbf{\Omega}}}^{\mathrm{H}} \boldsymbol{v}_{\overline{\mathbf{\Omega}}}\right) \\
& -2 \Re e\left(z_{\mathbf{\Omega}}^{0 \mathrm{H}} \boldsymbol{v}_{\mathbf{\Omega}}\right)+\lambda\left\|\boldsymbol{z}_{\mathbf{\Omega}}-\boldsymbol{z}_{\mathbf{\Omega}}^{0}-\lambda^{-1} \boldsymbol{v}_{\mathbf{\Omega}}\right\|_{2}^{2} \\
& -\lambda^{-1}\left\|\boldsymbol{v}_{\mathbf{\Omega}}\right\|_{2}^{2}-\lambda \eta^{2}
\end{aligned}
$$

where $\overline{\boldsymbol{\Omega}}$ denotes the complementary set of $\boldsymbol{\Omega}$. Minimizing $\mathcal{L}$ with respects to $(\boldsymbol{u}, w, \boldsymbol{z})$ gives the dual objective which equals $-2 \Re e\left(z_{\Omega}^{0 \mathrm{H}} v_{\Omega}\right)-\lambda^{-1}\left\|v_{\Omega}\right\|_{2}^{2}-\lambda \eta^{2}$ if $\mathrm{T}^{*}(\boldsymbol{W}-\boldsymbol{I})=0, s=1$, and $\boldsymbol{v}_{\overline{\mathbf{\Omega}}}=0$, otherwise $-\infty$, where $\mathrm{T}^{*}(\cdot)$ denotes the adjoint operator of $\mathrm{T}(\cdot)$. Noting $\left(\left\|v_{\boldsymbol{\Omega}}\right\|_{2}-\lambda \eta\right)^{2} \geq 0$, i.e. $\lambda^{-1}\left\|v_{\Omega}\right\|^{2}+\lambda \eta^{2} \geq 2 \eta\left\|v_{\Omega}\right\|_{2}$, so we obtain the dual problem (15) as the following form

$$
\begin{array}{ll}
\min _{\boldsymbol{v}, \boldsymbol{W}} \eta\left\|\boldsymbol{v}_{\mathbf{\Omega}}\right\|_{2}+\mathfrak{R} e\left(\boldsymbol{z}_{\mathbf{\Omega}}^{0 \mathrm{H}} \boldsymbol{v}_{\mathbf{\Omega}}\right), \\
\text { s.t. }\left[\begin{array}{ll}
1 & \boldsymbol{v}^{\mathrm{H}} \\
\boldsymbol{v} & \boldsymbol{W}
\end{array}\right] \geq 0, \boldsymbol{v}_{\overline{\mathbf{\Omega}}}=0, \mathrm{~T}^{*}(\boldsymbol{W}-\boldsymbol{I})=0 .
\end{array}
$$

\section{References}

[1] VAN TREES, H. L. Detection, Estimation, and Modulation Theory (Part IV: Optimum Array Processing). New York, USA: Wiley, 2004. ISBN: 0-471-09390-4

[2] PAN, Y., LUO, G. Q., JIN, H., et al. Direction-of-arrival estimation with ULA: A spatial annihilating filter reconstruction perspective. IEEE Access, 2018, vol. 6, p. 23172-23179. DOI: 10.1109/ACCESS.2018.2828799

[3] MOFFET, A. Minimum-redundancy linear arrays. IEEE Transactions on Antennas and Propagation, 1968, vol. 16, no. 2, p. 172-175. DOI: $10.1109 /$ TAP.1968.1139138 
[4] BLOOM, G. S., GOLOMB, S. W. Applications of numbered undirected graphs. Proceedings of the IEEE, 1977, vol. 65, no. 4, p. 562-570. DOI: $10.1109 /$ PROC.1977.10517

[5] VAIDYANATHAN, P. P., PAL, P. Sparse sensing with co-prime samplers and arrays. IEEE Transactions on Signal Processing, 2011, vol. 59, no. 2, p. 573-586. DOI: 10.1109/TSP.2010.2089682

[6] PAL, P., VAIDYANATHAN, P. P. Nested arrays: A novel approach to array processing with enhanced degrees of freedom. IEEE Transactions on Signal Processing, 2010, vol. 58, no. 8, p. 4167-4181. DOI: 10.1109/TSP.2010.2049264

[7] ABRAMOVICH, Y. I., SPENCER, N. K., GOROKHOV, A. Y. Positive-definite Toeplitz completion in DOA estimation for nonuniform linear antenna arrays. II. Partially augmentable arrays. IEEE Transactions on Signal Processing, 1999, vol. 47, no. 9, p. 1502-1521. DOI: $10.1109 / 78.765119$

[8] PAL, P., VAIDYANATHAN, P. P. Coprime sampling and the MUSIC algorithm. In Proceedings of 2011 Digital Signal Processing and Signal Processing Education Meeting (DSP/SPE). Sedona (AZ, USA), 2011, p. 289-294. DOI: 10.1109/DSPSPE.2011.5739227

[9] PAL, P., VAIDYANATHAN, P. P. A grid-less approach to underdetermined direction of arrival estimation via low rank matrix denoising. IEEE Signal Processing Letters, 2014, vol. 21, no. 6, p. 737-741. DOI: 10.1109/LSP.2014.2314175

[10] DONOHO, D. L. Compressed sensing. IEEE Transactions on Information Theory, 2006, vol. 52, no. 4, p. 1289-1306. DOI: 10.1109/TIT.2006.871582

[11] CANDES, E. J., ROMBERG, J., TAO, T. Robust uncertainty principles: Exact signal reconstruction from highly incomplete frequency information. IEEE Transactions on Information Theory, 2006, vol. 52, no. 2, p. 489-509. DOI: 10.1109/TIT.2005.862083

[12] ZHANG, Y. D., AMIN, M. G., HIMED, B. Sparsity-based DOA estimation using co-prime arrays. In Proceedings of 2013 IEEE International Conference on Acoustics, Speech and Signal Processing (ICASSP). Vancouver (BC, Canada), 2013, p. 3967-3971. DOI: 10.1109/ICASSP.2013.6638403

[13] PAN, Y., LUO, G. Q., JIN, H., et al. DOA estimation with planar array via spatial finite rate of innovation reconstruction. Signal Processing, 2018, vol. 153, p. 47-57. DOI: 10.1016/j.sigpro.2018.07.001

[14] TAN, Z., NEHORAI, A. Sparse direction of arrival estimation using co-prime arrays with off-grid targets. IEEE Signal Processing Letters, 2014, vol. 21, no. 1, p. 26-29. DOI: 10.1109/LSP.2013.2289740

[15] YANG, Z., LI, J., STOICA, P., et al. Sparse Methods for Direction-of Arrival Estimation. 65. [Online] Cited 2016-10-3. Available at: arXiv: 1609.09596 [cs.IT]

[16] YANG, Z., XIE, L. Exact joint sparse frequency recovery via optimization methods. IEEE Transactions on Signal Processing, 2016, vol. 64, no. 19, p. 5145-5157. DOI: 10.1109/TSP.2016.2576422

[17] YANG, Z., XIE, L. Enhancing sparsity and resolution via reweighted atomic norm minimization. IEEE Transactions on Signal Processing, 2016, vol. 64, no. 4, p. 995-1006. DOI: 10.1109/TSP.2015.2493987

[18] YANG, Z., XIE, L. On gridless sparse methods for line spectral estimation from complete and incomplete data. IEEE Transactions on Signal Processing, 2015, vol. 63, no. 12, p. 3139-3153. DOI: 10.1109/TSP.2015.2420541

[19] YANG, Z., XIE, L. Continuous compressed sensing with a single or multiple measurement vectors. In Proceedings of 2014 IEEE
Workshop on Statistical Signal Processing (SSP). Gold Coast (Australia), 2014, p. 288-291. DOI: 10.1109/SSP.2014.6884632

[20] GUO, M., CHEN, T., WANG, B. An improved DOA estimation approach using coarray interpolation and matrix denoising. Sensors, 2017, vol. 17, no. 5, p. 1-12. DOI: 10.3390/s17051140

[21] LIU, C., VAIDYANATHAN, P. P., PAL, P. Coprime coarray interpolation for DOA estimation via nuclear norm minimization. In Proceedings of 2016 IEEE International Symposium on Circuits and Systems (ISCAS). Montreal (QC, Canada), 2016, p. 2639-2642. DOI: 10.1109/ISCAS.2016.7539135

[22] ZHOU, C., GU, Y., FAN, X., et al. Direction-of-arrival estimation for coprime array via virtual array interpolation. IEEE Transactions on Signal Processing, 2018, vol. 66, no. 22, p. 5956-5971. DOI: 10.1109/TSP.2018.2872012

[23] LI, Y., CHI, Y. Off-the-grid line spectrum denoising and estimation with multiple measurement vectors. IEEE Transactions on Signal Processing, 2016, vol. 64, no. 5, p. 1257-1269. DOI: 10.1109/TSP.2015.2496294

[24] QIN, S., ZHANG, Y. D., AMIN, M. G. Generalized coprime array configurations for direction-of-arrival estimation. IEEE Transactions on Signal Processing, 2015, vol. 63, no. 6, p. 1377-1390. DOI: 10.1109/TSP.2015.2393838

[25] CHANDRASEKARAN, V., RECHT, B., PARRILO, P. A., et al. The convex geometry of linear inverse problems. Foundations of Computational Mathematics, 2012, vol. 12, no. 6, p. 805-849. DOI: $10.1007 / \mathrm{s} 10208-012-9135-7$

[26] TANG, G., BHASKAR, B. N., SHAH, P., et al. Compressed sensing off the grid. IEEE Transactions on Information Theory, 2013, vol. 59, no. 11, p. 7465-7490. DOI: 10.1109/TIT.2013.2277451

[27] BOYD, S. P., VANDENBERGHE, L. Convex Optimization. Cambridge, U.K.: Cambridge Univ. Press, 2004. ISBN: 978-0521-83378-3

[28] TOH, K.-C., TODD, M. J., TÜTÜNCÜ, R. H. SDPT3-a MATLAB software package for semidefinite programming version 1.3. Optimization Methods Software, 1999, vol. 11, no. 1-4, p. $545-581$. DOI: $10.1080 / 10556789908805762$

[29] LIU, C., VAIDYANATHAN, P. P. Remarks on the spatial smoothing step in coarray MUSIC. IEEE Signal Processing Letters, 2015, vol. 22, no. 9, p. 1438-1442. DOI: 10.1109/LSP.2015.2409153

[30] SCHMIDT, R. O. Multiple emitter location and signal parameter estimation. IEEE Transactions on Antennas and Propagation, 1986, vol. 34, no. 3, p. 276-280. DOI: 10.1109/TAP.1986.1143830

[31] ZHOU, C. ZHOU, J. Direction-of-arrival estimation with coarray ESPRIT for coprime array. Sensors, 2017, vol. 17, no. 8, p. 1-17. DOI: $10.3390 / \mathrm{s} 17081779$

[32] ROY, R., KAILATH, T. ESPRIT-estimation of signal parameters via rotational invariance techniques. IEEE Transactions on Acoustics, Speech, and Signal Processing, 1989, vol. 37, no. 7, p. $984-995$. DOI: $10.1109 / 29.32276$

[33] CHEN, H., HOU, C., ZHU, W. P., et al. ESPRIT-like twodimensional direction finding for mixed circular and strictly noncircular sources based on joint diagonalization. Signal Processing, 2017, vol. 141, p. 48-56. DOI: 10.1016/j.sigpro.2017.05.024

[34] RAO, B. D., HARI, K. V. S. Performance analysis of root-MUSIC In The Twenty-Second Asilomar Conference on Signals, Systems and Computers. Pacific Grove (CA, USA), 1988, p. 578-582. DOI: 10.1109/ACSSC.1988.754608

[35] LIU CONGFENG, LIAO GUISHENG. Fast algorithm for RootMUSIC with real-valued egendecomposition. In Proceedings of 
2006 CIE International Conference on Radar. Shanghai (China), 2006, p. 1-4. DOI: 10.1109/ICR.2006.343159

[36] GRANT, M., BOYD, S. CVX: Matlab Software for Disciplined Convex Programming. [Online] Cited 2014-3. Available at: http://cvxr.com/cvx.

[37] LIU, C. L., VAIDYANATHAN, P. P. Cramér-Rao bounds for coprime and other sparse arrays, which find more sources than sensors. Digital Signal Processing, 2016, vol. 61, p. 43-61. DOI: 10.1016/j.dsp.2016.04.011

\section{About the Authors ...}

Yujian PAN was born in 1987. He received his Ph.D. from the National University of Defense Technology in 2015. He is currently a Lecturer with the School of Electronics and Information, Hangzhou Dianzi University, Hangzhou, China. His main research interests include microwave circuit design, radar system design, radar signal processing, array signal processing, and machine learning. From 2019 to 2020, he is a visiting scholar at the Dept. of Electrical Engineering (ESAT), KU Leuven, Belgium.

Min YAO was born in 1996. She received a B.E. degree in Taiyuan University of Science and Technology, Taiyuan, China, in 2018. She is now studying for M.S. degree in Electronic Science and Technology at Hangzhou Dianzi
University. Her current research interest lies in the area of array signal processing.

Guo Qing LUO (corresponding author) was born in 1979. $\mathrm{He}$ received the Ph.D. degree from the Southeast University, Nanjing, China, in 2007. Since 2007, he has been a Lecturer with the Faculty of School of Electronics and Information, Hangzhou Dianzi University, Hangzhou, China, and was promoted to Professor in 2011. His current research interests include RF, microwave and mm-wave passive devices, antenna array, and frequency selective surfaces.

Bai Cao PAN was born in 1989. He received the Ph.D. degree in Electrical Engineering from the Southeast University, Nanjing, China in 2018. He joined the School of Electronics and Information, Hangzhou Dianzi University, where he is currently an Associate Professor. His research interests include engineered electromagnetic structures, metamaterials, spoof surface plasmon polaritons, antennas array, and microwave circuits.

Xiaoxin GAO was born in 1995. She received a B.E. degree in Shijiazhuang University, Shijiazhuang, China, in 2019. She is now studying for M.S. degree in Electronics and Communication Engineering at Hangzhou Dianzi University. Her current research interest is the array signal processing. 